\title{
THE BUDAN-FOURIER THEOREM AND HERMITE-BIRKHOFF SPLINE INTERPOLATION
}

BY

\author{
T. N. T. GOODMAN AND S. L. LEE
}

\begin{abstract}
We extend the classical Budan-Fourier theorem to Hermite-Birkhoff splines, that is splines whose knots are determined by a finite incidence matrix. This is then applied to problems of interpolation by Hermite-Birkhoff splines, where the nodes of interpolation are also determined by a finite incidence matrix. For specified knots and nodes in a finite interval, conditions are examined under which there is a unique interpolating spline for any interpolation data. For knots and nodes spaced periodically on the real line, conditions are examined under which there is a unique interpolating spline of power growth for data of power growth.
\end{abstract}

1. Introduction. By an incidence matrix we shall mean a matrix whose entries are zero or one. Take incidence matrices

$$
E=\left\|E_{i j}\right\|_{i=0 j=0}^{m} \quad \text { and } \quad F=\left\|F_{i j}\right\|_{i=0}^{m}{ }_{j=0}^{n}
$$

and let $x=\left(x_{0}, \ldots, x_{m}\right), 0=x_{0}<x_{1}<\cdots<x_{m}=1$.

We define $\zeta(F, x):=\left\{f:[0,1] \rightarrow \mathbf{C} ; f \mid\left(x_{i}, x_{i+1}\right) \in \pi_{n}, i=0, \ldots, m-1\right.$, and $f^{(n-j)}\left(x_{i}^{-}\right)=f^{(n-j)}\left(x_{i}^{+}\right), \forall(i, j)$ with $0<i<m$ and $\left.F_{i j}=0\right\}$, where $\pi_{n}$ denotes the set of polynomials of degree $\leqslant n$.

For $j \geqslant 0$ we write

$$
f^{(j)}(0)=f^{(j)}\left(0^{+}\right), \quad f^{(j)}(1)=f^{(j)}\left(1^{-}\right),
$$

and

$$
f^{(j)}\left(x_{i}\right)=\frac{1}{2}\left\{f^{(j)}\left(x_{i}^{-}\right)+f^{(j)}\left(x_{i}^{+}\right)\right\} \quad \text { for } 0<i<m .
$$

We shall refer to the following interpolation problem as the I.P. $(E, F, x)$.

$$
\left\{\begin{array}{l}
\text { For numbers }\left\{y_{i j} ; E_{i j}=1\right\}, \text { find } f \in \zeta(F, x) \\
\text { satisfying } f^{(j)}\left(x_{i}\right)=y_{i j} .
\end{array}\right.
$$

We say the I.P. $(E, F, x)$ is poised if for any choice of $\left\{y_{i j}\right\},(1.1)$ has a unique solution.

The case of interpolation by polynomials, i.e. $0<i<m \Rightarrow F_{i j}=0$, has been studied extensively. The study was initiated by G. D. Birkhoff [2] and later revived by Schoenberg [16] who called this problem Hermite-Birkhoff (HB) interpolation and introduced the notion of an incidence matrix.

Received by the editors February 20, 1980 and, in revised form, March 23, 1981.

1980 Mathematics Subject Classification. Primary 41A15, 41A05.

(C)1982 American Mathematical Society 0002-9947/82/0000-1044/\$05.25 
Next suppose $(E, F, x)$ are as above with the extra condition that

$$
E_{0 j}=E_{m j} \quad \text { and } \quad F_{0 j}=F_{m j}, \quad j=0, \ldots, n .
$$

We define $\mathcal{E}(F, x):=\left\{f: \quad \mathbf{R} \rightarrow \mathbf{C} ; \quad \forall \nu \in \mathbf{Z}, f \mid\left(\nu+x_{i}, \nu+x_{i+1}\right) \in \pi_{n}, \quad i=\right.$ $0, \ldots, m-1$, and $f^{(n-j)}\left(\nu+x_{i}^{-}\right)=f^{(n-j)}\left(\nu+x_{i}^{+}\right) \forall(i, j)$ with $\left.F_{i j}=0\right\}$.

For any $\nu, i, j$ we write

$$
f^{(j)}\left(\nu+x_{i}\right)=\frac{1}{2}\left\{f^{(j)}\left(\nu+x_{i}^{-}\right)+f^{(j)}\left(\nu+x_{i}^{+}\right)\right\} .
$$

We shall refer to the following 'cardinal' interpolation problem as the C.I.P. $(E, F, x)$.

$$
\left\{\begin{array}{l}
\text { For sequences of numbers }\left\{y^{(i, j)}\right\}=\left\{\left(y_{\nu}^{(i, j)}\right)_{\nu=-\infty}^{\infty} ; 0 \leqslant i<m,\right. \\
\text { and } \left.E_{i j}=1\right\}, \text { find } f \in \Theta(F, x) \text { satisfying } f^{(j)}\left(\nu+x_{i}\right)=y_{\nu}^{(i, j)} .
\end{array}\right.
$$

In [10] Lipow and Schoenberg considered the C.I.P. $(E, F, x)$ for the following case: $m=1, n$ is odd, $E=F$ and for some $1 \leqslant r \leqslant \frac{1}{2}(n+1)$,

$$
E_{0 j}=E_{1 j}= \begin{cases}1, & j=0, \ldots, r-1, \\ 0, & j=r, \ldots, n .\end{cases}
$$

In this case $\varrho(F, x)$ comprises cardinal Hermite splines with knots of multiplicity $r$ at the integers. The problem (1.2) then requires interpolation of the spline and its first $(r-1)$ derivates at the integers, and is called cardinal Hermite interpolation. In this case it is proved in [10] that the C.I.P. is 'poised' in the following sense.

We say the C.I.P. $(E, F, x)$ is poised if the following holds. Suppose $\left\{y^{(i, j)}\right\}$ have power growth, i.e. there is a number $\gamma$ with $y_{\nu}^{(i, j)}=O\left(|\nu|^{\gamma}\right)$ as $\nu \rightarrow \pm \infty, \forall(i, j)$. Then (1.2) has a unique solution of power growth of the same order, i.e. $f(x)=$ $O\left(|x|^{\gamma}\right)$ as $x \rightarrow \pm \infty$.

In $\$ 2$ of this paper we derive an extension of the classical Budan-Fourier theorem to elements of $\zeta(f, x)$, which is then applied in $\S \S 3$ and 4 , where we investigate conditions under which problems (1.1) and (1.2) are poised. The work of $\S \S 2$ and 4 is further exploited in another paper of the authors [6].

2. The Budan-Fourier theorem for $\mathrm{HB}$ splines. For any incidence matrix $F=$ $\left\|F_{i j}\right\|_{i=0 j=0}^{m}$ and $x=\left(x_{0}, \ldots, x_{m}\right), \quad a=x_{0}<\cdots<x_{m}=b$, we define $\zeta_{0}(F):=\zeta_{0}(F, x):=\left\{f: \mathbf{R} \rightarrow \mathbf{R} ; f \mid\left(x_{i}, x_{i+1}\right) \in \pi_{n}, i=0, \ldots, m-1, f(x)=0\right.$ for $x \notin[a, b]$ and $f^{(n-j)}\left(x_{i}^{-}\right)=f^{(n-j)}\left(x_{i}^{+}\right), \forall(i, j)$ with $\left.F_{i j}=0\right\}$.

If $f$ is an element of $\zeta_{0}(F)$ for some $F$, we say $f$ is an HB spline.

By a block in $F$ we mean a sequence $\{(i, j)\}, j=k, \ldots, k+l-1$, with $F_{i j}=1$, $k \leqslant j \leqslant k+l-1$, and $F_{i(k-1)} \neq 1 \neq F_{i(k+l)}$. The block is called even or odd as $l$ is even or odd. We say the block is supported if $\exists i_{1}, i_{2}, j_{1}, j_{2}$ with $i_{1}<i<i_{2}, j_{1}, j_{2}<k$ and $F_{i_{1} j_{1}}=F_{i_{2} j_{2}}=1$. We let $b(F)$ denote the number of supported odd blocks in $F$.

Both Lorentz [11] and Schumaker [17] have derived bounds of the following form for the number of zeros of $\mathrm{HB}$ splines. If $f \in \zeta_{0}(F)$ has exact degree $n$ (that is $f^{(n+1)}$ is the zero function and $f^{(n)}$ is not the zero function), then

$$
Z(f)<\sum_{i=0}^{m} \sum_{j=0}^{n} F_{i j}-(n+1)+b(F)
$$


where $Z(f)$ denotes the number of zeros of $f$ in $(a, b)$ counted with multiplicity in some specified manner. These results were an improvement of a similar result by Ferguson [4] and indeed an estimate of this type was given much earlier by Birkhoff [2].

We now recall the classical Budan-Fourier theorem which gives a bound for the number of zeros of a polynomial in an interval $(a, b)$. For a real vector $v=$ $\left(v_{0}, \ldots, v_{k}\right)$, let $S^{-} v$ (or $S^{+} v$ ) denote the minimal (or maximal) number of sign changes in the sequence $v$ achievable by appropriate assignment of signs to the zero entries of $v$. If $S^{-} v=S^{+} v$, we denote their common value by $S v$. Then the Budan-Fourier theorem states that if $p$ is a polynomial of exact degree $n$, then

$$
Z(p) \leqslant S^{-}\left(p(a), \ldots, p^{(n)}(a)\right)-S^{+}\left(p(b), \ldots, p^{(n)}(b)\right),
$$

where $Z(p)$ denotes the number of zeros of $p$ in $(a, b)$ counted with multiplicity in the usual way. This result has been generalised by Melkman [12] and by deBoor and Schoenberg [3] to give a bound for the number of zeros of Hermite splines, that is HB splines where for $i=1, \ldots, m-1$, there is a number $K_{i}$ such that $F_{i j}=1$ iff $j<K_{i}$.

We shall prove a result of Budan-Fourier type for HB splines which generalises both (2.1) and (2.2). First we must describe how to count the zeros of an HB spline $f$. For any number $c$ we shall write

$$
f(c)^{-}\left(\text {or } f(c)^{+}\right)=\left\{\begin{array} { l } 
{ - 1 , } \\
{ 0 , } \\
{ 1 , }
\end{array} \text { if } f ( x ) \left\{\begin{array}{l}
<0 \\
=0, \\
>0
\end{array}\right.\right.
$$

on $(c-\delta, c)($ or $(c, c+\delta))$ for some $\delta>0$.

Now suppose we have numbers $\alpha \leqslant \beta$ such that $f(x)=0$ on $(a, \beta)$ and $f(\alpha)^{-} \neq 0$ $\neq f(\beta)^{+}$. Define $l \geqslant 0, r \geqslant 0$ by $f\left(\alpha^{-}\right)=\cdots=f^{(l-1)}\left(\alpha^{-}\right)=0, f^{(l)}\left(\alpha^{-}\right) \neq 0$, $f\left(\beta^{+}\right)=\cdots=f^{(r-1)}\left(\beta^{+}\right)=0, f^{(r)}\left(\beta^{+}\right) \neq 0$. Let $s=\min (l, r)$. Then we say $[\alpha, \beta]$ is a zero of $f$ of multiplicity $M$, where

$$
M= \begin{cases}s, & \text { if } \alpha=\beta \text { and } f(\alpha)^{-} f(\alpha)^{+}=(-1)^{s}, \\ s+1, & \text { otherwise. }\end{cases}
$$

If $\alpha=\beta$ is not a knot (i.e. not an element of $x$ ), then this is the usual multiplicity. We say $[\alpha, \beta]$ is a continuous zero if $s \geqslant 1$, i.e. $f\left(\alpha^{-}\right)=f\left(\beta^{+}\right)=0$. Finally we let $Z(f)$ denote the total number of zeros of $f$ counted with multiplicity. This zero count is slightly stronger than those considered by Lorentz [11] and Schumaker [17].

THEOREM 2.1. If $f \in \zeta_{0}(F)$ has exact degree $n$, then

$$
\begin{aligned}
Z(f) \leqslant & S^{-}\left(f\left(a^{+}\right), \ldots, f^{(n)}\left(a^{+}\right)\right)-S^{+}\left(f\left(b^{-}\right), \ldots, f^{(n)}\left(b^{-}\right)\right) \\
& +\sum_{i=1}^{m-1} \sum_{j=0}^{n} F_{i j}+b(F) .
\end{aligned}
$$

Our proof uses both the approach of [3] and that introduced in [4], which is used also in $[\mathbf{1 1}, \mathbf{1 7}]$. We shall need the following lemma which is essentially Lemma 1.2 of Karlin and Micchelli [8]. 
Lemma 2.1. If $f \in C^{n}[0, \delta]$ and $f^{(n)}\left(0^{+}\right) \neq 0$, then

$$
f^{(j)}(0)^{+} \neq 0, \quad j=0, \ldots, n,
$$

and

$$
S^{-}\left(f\left(0^{+}\right), \ldots, f^{(n)}\left(0^{+}\right)\right)=S\left(f(0)^{+}, \ldots, f^{(n)}(0)^{+}\right) .
$$

Similarly if $f \in C^{n}[-\delta, 0]$ and $f^{(n)}\left(0^{-}\right) \neq 0$, then

$$
f^{(j)}(0)^{-} \neq 0, \quad j=0, \ldots, n,
$$

and

$$
S^{+}\left(f\left(0^{-}\right), \ldots, f^{(n)}\left(0^{-}\right)\right)=S\left(f(0)^{-}, \ldots, f^{(n)}(0)^{-}\right) .
$$

Proof of TheOrem 2.1. For any HB spline $f$, we let $\left[a_{f}, b_{f}\right]$ denote the support of $f$, i.e. $f(x)=0$ outside $\left[a_{f}, b_{f}\right]$ and $f\left(a_{f}\right)^{+} \neq 0 \neq f\left(b_{f}\right)^{-}$. We shall classify a point $\gamma$ at which $f$ has a discontinuity as follows.

Type 1. $f(\gamma)^{-} f(\gamma)^{+}>0$.

Type 2. $f(\gamma)^{-} f(\gamma)^{+}<0$ and $f^{\prime}(\gamma)^{-} f^{\prime}(\gamma)^{+}<0$.

Type 3. $f(\gamma)^{-} f(\gamma)^{+}<0$ and $f^{\prime}(\gamma)^{-} f^{\prime}(\gamma)^{+}>0$.

Type 4. $\gamma=a_{f}$.

Type 5. $\gamma=b_{f}$.

Type 6. Otherwise.

For $i=1, \ldots, 6$, we let $d_{i}$ denote the number of discontinuities of $f$ of type $i$. We shall show that if $f$ is any HB spline and $f^{\prime}$ is not the zero function, then

$$
\begin{aligned}
Z(f) \leqslant & Z\left(f^{\prime}\right)+S\left(f\left(a_{f^{\prime}}\right)^{+}, f^{\prime}\left(a_{f^{\prime}}\right)^{+}\right) \\
& -S\left(f\left(b_{f^{\prime}}\right)^{-}, f^{\prime}\left(b_{f^{\prime}}\right)^{-}\right)+2 d_{3}+d_{6} .
\end{aligned}
$$

Suppose $f$ has continuous zeros at $I_{i}=\left[\alpha_{i}, \beta_{i}\right]$ of multiplicity $m_{i}, i=1, \ldots, N$, where $I_{1}<I_{2}<\cdots<I_{N}$. We note that $a_{f^{\prime}}<I_{1} \leqslant I_{N}<b_{f^{\prime}}$ since $f$ is a step function outside $\left[a_{f^{\prime}}, b_{f^{\prime}}\right]$. Thus $f^{\prime}$ has zeros at $I_{i}$ of multiplicity $m_{i}-1, i=1, \ldots, N$.

For any $i=1, \ldots, N-1, f f^{\prime}$ changes sign in $\left(\beta_{i}, \alpha_{i+1}\right)$. Also if $f\left(a_{f^{\prime}}\right)^{+} f^{\prime}\left(a_{f^{\prime}}\right)^{+}=1$, then $f f^{\prime}$ changes sign in $\left(a_{f^{\prime}}, \alpha_{1}\right)$, and if $f\left(b_{f^{\prime}}\right)^{-} f^{\prime}\left(b_{f^{\prime}}\right)^{-}=-1$, then $f f^{\prime}$ changes sign in $\left(\beta_{n}, b_{f^{\prime}}\right)$. Thus there are at least $v$ intervals in which $f f^{\prime}$ changes sign, where

$$
v=N-S\left(f\left(a_{f^{\prime}}\right)^{+}, f^{\prime}\left(a_{f^{\prime}}\right)^{+}\right)+S\left(f\left(b_{f^{\prime}}\right)^{-}, f^{\prime}\left(b_{f^{\prime}}\right)^{-}\right) \text {. }
$$

Now if $f f^{\prime}$ changes sign in an open interval $J$, there are 3 possibilities.

(a) $f^{\prime}$ vanishes on $[\alpha, \beta] \subset J$ with $\alpha<\beta$.

(b) $\exists \gamma \in J$ with $f(\gamma)^{-} f(\gamma)^{+}>0$ and $f^{\prime}(\gamma)^{-} f^{\prime}(\gamma)^{+}<0$.

(c) $\exists \gamma \in J$ with $f(\gamma)^{-} f(\gamma)^{+}<0$ and $f^{\prime}(\gamma)^{-} f^{\prime}(\gamma)^{+}>0$, i.e. $\gamma$ is a discontinuity of $f$ of Type 3 .

Thus there are at least $v=d_{3}$ zeros of $f^{\prime}$ of type (a) or (b). In addition we have at least $d_{2}$ zeros of $f^{\prime}$ at discontinuities of $f$ of Type 2 . So we have

$$
Z\left(f^{\prime}\right) \geqslant \sum_{i=1}^{N}\left(m_{i}-1\right)+v-d_{3}+d_{2} .
$$


But $Z(f) \leqslant \sum_{i=1}^{N} m_{i}+d_{2}+d_{3}+d_{6}$. So $Z\left(f^{\prime}\right) \geqslant Z(f)-\left(d_{2}+d_{3}+d_{6}\right)-N+v$ $-d_{3}+d_{2}$, which gives (2.3).

Now if $f$ is continuous at $a_{f}$, then $a_{f}=a_{f^{\prime}}$ and $S\left(f\left(a_{f}\right)^{+}, f^{\prime}\left(a_{f}\right)^{+}\right)=0$. Also if $f$ is continuous at $b_{f}$, then $b_{f}=b_{f^{\prime}}$ and $S\left(f\left(b_{f}\right)^{-}, f^{\prime}\left(b_{f^{\prime}}\right)^{-}\right)=1$. So we also have

$$
\begin{gathered}
Z(f) \leqslant Z\left(f^{\prime}\right)-S\left(f\left(b_{f^{\prime}}\right)^{-}, f^{\prime}\left(b_{f^{\prime}}\right)^{-}\right)+d_{4}+2 d_{3}+d_{6}, \\
Z(f) \leqslant Z\left(f^{\prime}\right)+S\left(f\left(a_{f^{\prime}}\right)^{+}, f^{\prime}\left(a_{f^{\prime}}\right)^{+}\right)-1+d_{5}+2 d_{3}+d_{6}, \\
Z(f) \leqslant Z\left(f^{\prime}\right)-1+d_{4}+d_{5}+2 d_{3}+d_{6} .
\end{gathered}
$$

Now take an incidence matrix $F=\left\|F_{i j}\right\|_{i=0 j=0}^{m}$ and $f \in \zeta_{0}(F, x)$ of exact degree $n$. We define matrices $G=\left\|G_{i j}\right\|_{i=0 j=0}^{m}$ and $H=\left\|H_{i j}\right\|_{i=0 j=0}^{m}$ by

$$
G_{i j}= \begin{cases}1, & \text { if } x_{i} \text { is a discontinuity of } f^{(n-j)}, \\ 0, & \text { otherwise }\end{cases}
$$

and

$$
H_{i j}= \begin{cases}2, & \text { if } x_{i} \text { is a discontinuity of } f^{(n-j)} \text { of Type } 3, \\ 1, & \text { if } x_{i} \text { is a discontinuity of } f^{(n-j)} \text { of Type } 4,5 \text { or } 6, \\ 0, & \text { otherwise. }\end{cases}
$$

Let $p$ and $q$ be the largest integers with $a_{f^{(p)}}=a$ and $b_{f^{(q)}}=b$, and assume $p<q<n$. Then by repeated application of (2.3) we have

$$
\begin{aligned}
Z(f) \leqslant & Z\left(f^{(p)}\right)+S\left(f(a)^{+}, \ldots, f^{(p)}(a)^{+}\right) \\
& -S\left(f(b)^{-}, \ldots, f^{(p)}(b)^{-}\right)+\sum_{i=1}^{m-1} \sum_{j=n-p+1}^{n} H_{i j} .
\end{aligned}
$$

Then by repeated application of (2.4) we have

$$
\begin{aligned}
Z\left(f^{(p)}\right) \leqslant & Z\left(f^{(q)}\right)-S\left(f^{(p)}(b)^{-}, \ldots, f^{(q)}(b)^{-}\right) \\
& +\sum_{i=1}^{m-1} \sum_{j=n-q+1}^{n-p} H_{i j}+1 .
\end{aligned}
$$

Finally repeated application of (2.6) gives

$$
Z\left(f^{(q)}\right) \leqslant Z\left(f^{(n)}\right)-(n-q)+\sum_{i=1}^{m-1} \sum_{j=1}^{n-q} H_{i j}+1 .
$$

Combining (2.7), (2.8) and (2.9) gives

$$
\begin{aligned}
Z(f) \leqslant & Z\left(f^{(n)}\right)+S\left(f(a)^{+}, \ldots, f^{(p)}(a)^{+}\right) \\
& -S\left(f(b)^{-}, \ldots, f^{(q)}(b)^{-}\right)+\sum_{i=1}^{m-1} \sum_{j=1}^{n} H_{i j}-(n-q)+2 .
\end{aligned}
$$

But by Lemma 2.1,

$$
\begin{aligned}
S\left(f(a)^{+}, \ldots, f^{(p)}(a)^{+}\right) & =S^{-}\left(f\left(a^{+}\right), \ldots, f^{(p)}\left(a^{+}\right)\right) \\
& =S^{-}\left(f\left(a^{+}\right), \ldots, f^{(n)}\left(a^{+}\right)\right)
\end{aligned}
$$


and

$$
\begin{aligned}
S\left(f(b)^{-}, \ldots, f^{(q)}(b)^{-}\right)+n-q & =S^{+}\left(f\left(b^{-}\right), \ldots, f^{(q)}\left(b^{-}\right)\right)+n \\
& =S^{+}\left(f\left(b^{-}\right), \ldots, f^{(n)}\left(b^{-}\right)\right) .
\end{aligned}
$$

Also $Z\left(f^{(n)}\right) \leqslant \sum_{i=1}^{m-1} H_{i 0}-2$.

Thus (2.10) gives

$$
\begin{aligned}
Z(f) \leqslant & \sum_{i=1}^{m-1} \sum_{j=0}^{n} H_{i j}+S^{-}\left(f\left(a^{+}\right), \ldots, f^{(n)}\left(a^{+}\right)\right) \\
& -S^{+}\left(f\left(b^{-}\right), \ldots, f^{(n)}\left(b^{-}\right)\right) .
\end{aligned}
$$

We can similarly deduce (2.11) for other values of $p$ and $q$ by suitable application of (2.3)-(2.6).

Now suppose $H_{i j}=2$. Then if $G_{i(j-1)}=1, x_{i}$ is a discontinuity of $f^{(n-j+1)}$ of Type 1 and so $H_{i(j-1)}=0$. Also if $G_{i(j+1)}=1, x_{i}$ is a discontinuity of $f^{(n-j-1)}$ of Type 1 or 2 and so $H_{i(j+1)}=0$. Also $f^{(n-j+1)}\left(x_{i}\right)^{-} \neq 0$ and so $i_{1}<i$ and $j_{1}<j$ with $G_{i_{1} j_{1}}=1$. Similarly $\exists i_{2}>i$ and $j_{2}<j$ with $G_{i_{2} j_{2}}=1$.

Now suppose that for some $i, 1<i<m,\{(i, j)\}, j=k, \ldots, k+l-1$ is a block in $G$. Then if $\sum_{j=k}^{k+l-1} H_{i j}>1$, it must be that $\left\{H_{i k}, \ldots, H_{i(k+l-1)}\right\}$ is of the form $\{2,0,2, \ldots, 0,2\}$. Thus $l$ must be odd and the block must be supported. Hence

$$
\sum_{i=1}^{m-1} \sum_{j=0}^{n} H_{i j} \leqslant \sum_{i=1}^{m-1} \sum_{j=0}^{n} G_{i j}+b(G) .
$$

But since $G_{i j}=1 \Rightarrow F_{i j}=1$, it is easy to see that

$$
\sum_{i=1}^{m-1} \sum_{j=0}^{n} G_{i j}+b(G) \leqslant \sum_{i=1}^{m-1} \sum_{j=0}^{n} F_{i j}+b(F) .
$$

The result then follows from (2.11), (2.12) and (2.13).

We can immediately deduce the following.

COROllaRy 2.1. If $f \in \zeta_{0}(f)$ has exact degree $n$, then

$$
Z(f)<\sum_{i=0}^{m} \sum_{j=0}^{n} F_{i j}-(n+1)+b(F) .
$$

3. The interpolation problem $(E, F, x)$. We now consider the I.P. $(E, F, x)$ as in (1.1). Since the problem is independent of the values of $F_{0 j}$ and $F_{m j}, j=0, \ldots, n$, we may assume, without loss of generality

$$
F_{i j}=1 \quad \text { iff } E_{i(n-j)}=0, \quad i=0 \text { or } m, j=0, \ldots, n .
$$

For $(E, F, x)$ to be poised we require the number of interpolation conditions $\sum_{i, j} E_{i j}$ to equal the dimension of $\zeta(F, x)$, namely $n+1+\Sigma\left\{f_{i j}: 0<i<m, 0 \leqslant j \leqslant\right.$ $n\}$. This condition can be written neatly by defining, for any matrix $A=\left\|A_{i j}\right\|_{i=0}^{m}{ }_{j=0}^{n}$,

$$
M(A)=\sum_{j=0}^{n}\left\{\frac{1}{2} A_{0 j}+\sum_{i=1}^{m-1} A_{i j}+\frac{1}{2} A_{m j}\right\} .
$$


The above condition then becomes

$$
M(E)=M(F)
$$

and we henceforth assume this is satisfied. Then the I.P. $(E, F, x)$ is poised if and only if the only function $f$ in $\zeta(F, x)$ satisfying

$$
f^{(j)}\left(x_{i}\right)=0 \text { for all } i, j \text { with } E_{i j}=1
$$

is the zero function.

Our first result reveals a duality between the matrices $E$ and $F$. Since the original submission of this work, we discovered this had already been proved by Jetter [7], but we retain our proof for completeness.

THEOREM 3.1. The I.P. $(E, F, x)$ is poised if and only if the I.P. $(F, E, x)$ is poised.

Proof. By (3.1) and (3.2)

$$
\sum_{i=0}^{m-1} \sum_{j=0}^{n} F_{i j}=\sum_{i=1}^{m} \sum_{j=0}^{n} E_{i j}
$$

and we denote their common value by $N$. Let $\left\{\left(i_{r}, j_{r}\right) ; r=1, \ldots, N\right\}$ denote the elements of $\left\{(i, j) ; 0<i<m, F_{i j}=1\right\}$ ordered so that for $1<r<N, i_{r}<i_{r+1}$ or $i_{r}=i_{r+1}$ and $j_{r}<j_{r+1}$. Let $\left\{\left(k_{s}, l_{s}\right) ; s=1, \ldots, N\right\}$ denote the elements of $\{(i, j)$; $\left.0<i<m, E_{i j}=1\right\}$ ordered so that for $1 \leqslant s<N, k_{s}>k_{s+1}$ or $k_{s}=k_{s+1}$ and $l_{s}<l_{s+1}$.

Then any $f \in \zeta_{0}(F, x)$ can be written as

$$
f(x)=\sum_{r=1}^{N} \frac{\lambda_{r}\left(x-x_{i_{r}}\right)_{+}^{n-j_{r}}}{\left(n-j_{r}\right) !}
$$

for constant $\lambda_{1}, \ldots, \lambda_{N}$, where for any $x$ and $p$,

$$
x_{+}^{p}= \begin{cases}x^{p}, & x>0 \\ \frac{1}{2} x^{p}, & x=0 \\ 0, & x<0\end{cases}
$$

With the convention that $(p !)^{-1}=0$ for $p<0$, the conditions (3.3) become

$$
\sum_{r=1}^{N} \frac{\lambda_{r}\left(x_{k_{s}}-x_{i_{r}}\right)_{+}^{n-j_{r}-l_{s}}}{\left(n-j_{r}-l_{s}\right) !}=0, \quad s=1, \ldots, N
$$

Thus the I.P. $(E, F, x)$ is poised iff the matrix

$$
A=\left\|\frac{\left(x_{k_{s}}-x_{i_{r}}\right)_{+}^{n-j_{r}-l_{s}}}{\left(n-j_{r}-l_{s}\right) !}\right\|_{s, r=1}^{N}
$$

is nonsingular.

Defining $\hat{E}, \hat{F}, \hat{x}$ by $\hat{E}_{i j}=E_{(m-i) j}, \hat{F}_{i j}=F_{(m-i) j}, \hat{x}_{i}=1-x_{m-i}$, we see that the matrix corresponding to $A$ for the I.P. $(\hat{F}, \hat{E}, \hat{x})$ is the transpose of $A$. So the I.P. $(E, F, x)$ is poised iff the I.P. $(\hat{F}, \hat{E}, \hat{x})$ is poised. But by making the transformation $x \rightarrow 1-x$, we see the I.P. $(\hat{F}, \hat{E}, \hat{x})$ is poised iff the I.P. $(F, E, x)$ is poised. 
It will be convenient to introduce the matrix $G=\left\|G_{i j}\right\|_{i=0 j=0}^{m}{ }_{j=0}^{n}$ defined by $G_{i j}=$ $E_{i j}-F_{i(n-j)}$.

We note that (3.1) and (3.2) can then be rewritten as

$$
\begin{gathered}
G_{i j} \neq 0, \quad i=0 \text { or } m, j=0, \ldots, n, \\
M(G)=0 .
\end{gathered}
$$

We shall also let $|S|$ denote the cardinality of a set $S$, and for any matrix $A$ we write

$$
A\left(\begin{array}{c}
\mu_{1}, \ldots, \mu_{k} \\
\nu_{1}, \ldots, \nu_{l}
\end{array}\right):=\left\|A_{\mu, \nu_{l}}\right\|_{i=1 j=1}^{k},
$$

that is the submatrix of $A$ determined by the rows $\mu_{1}, \ldots, \mu_{k}$ and columns $\nu_{1}, \ldots, \nu_{l}$.

We now consider further conditions on $(E, F)$ which are necessary for the I.P. $(E, F, x)$ to be poised. First consider the case of interpolation by polynomials, i.e. $F_{i j}=0$ whenever $0<i<m$. If, for any $r=0, \ldots, n$,

$$
\sum_{i=0}^{m} \sum_{j=0}^{r} E_{i j}<\operatorname{dim} \pi_{r}
$$

then there would be a nonzero $f \in \pi_{r}$ satisfying (3.3) and the I.P. ( $\left.E, F, x\right)$ would not be poised. Thus we have the necessary conditions,

$$
\sum_{i=0}^{m} \sum_{j=0}^{r} E_{i j} \geqslant r+1, \quad r=0, \ldots, n \text {. }
$$

These are well-known Pólya conditions on $E$ (e.g. see [1]).

Next consider a general matrix $F$ and take any $0 \leqslant r \leqslant n, 0 \leqslant k<l \leqslant m$. Let $V$ denote the space of restrictions to $\left(x_{k}, x_{l}\right)$ of all functions in $(F, x)$ with exact degree $\leqslant r$. If

$$
\sum_{i=k}^{l} \sum_{j=0}^{r} E_{i j}+\mid\left\{(i, j) ; i=k \text { or } l, j=0, \ldots, r, E_{i j}=F_{i(n-j)}=0\right\} \mid<\operatorname{dim} V,
$$

then there would be a nonzero $g \in V$ which could be extended to a function $f \in \zeta(F, x)$ vanishing outside $\left[x_{k}, x_{l}\right]$ and satisfying (3.3). Thus for the I.P. $(E, F, x)$ to be poised we must have

$$
\begin{gathered}
\sum_{i=k}^{l} \sum_{j=0}^{r} E_{i j}+\mid\left\{(i, j) ; i=k \text { or } l, j=0, \ldots, r, E_{i j}=F_{i(n-j)}=0\right\} \mid \\
\geqslant r+1+\sum_{i=k+1}^{l-1} \sum_{j=0}^{r} F_{i(n-j)} .
\end{gathered}
$$

This can be written more neatly as

$$
\frac{1}{2} \mid\left\{(i, j) ; i=k \text { or } l, j=0, \ldots, r, G_{i j}=0\right\} \mid+M\left(G\left(\begin{array}{c}
k, \ldots, l \\
0, \ldots, r
\end{array}\right)\right) \geqslant 0 .
$$


By Theorem 3.1 we must also have

$$
\begin{gathered}
\frac{1}{2} \mid\left\{(i, j) ; i=k \text { or } l, j=n-r, \ldots, n, G_{i j}=0\right\} \mid \\
-M\left(G\left(\begin{array}{c}
k, \ldots, l \\
n-r, \ldots, n
\end{array}\right)\right) \geqslant 0 .
\end{gathered}
$$

These conditions have been considered by various authors in various forms and under various names, [7, 13, 15]. If $(E, F)$ satisfies (3.4) and (3.5) for any $0 \leqslant r \leqslant n$, $0 \leqslant k<l \leqslant m$, we shall say $(E, F)$ satisfies the Pólya conditions.

We next turn out attention to conditions on $(E, F)$ that are sufficient for the I.P. $(E, F, x)$ to be poised for any $x$. For the case when $0<i<m \Rightarrow F_{i j}=0$, there is a well-known result by Atkinson and Sharma [1] which states that the I.P. $(E, F, x)$ is poised if $E$ satisfies the Pólya conditions and has no supported odd blocks. This we proceed to generalise.

Following standard terminology we say an incidence matrix $E=\left\|E_{i j}\right\|_{i=0 j=0}^{m}$ is quasi-Hermite if for $i=1, \ldots, m-1$ there is a number $M_{i} \geqslant 0$ such that $E_{i j}=1$ iff $j<M_{i}$.

Theorem 3.2. Suppose $(E, F)$ satisfies the Pólya conditions and $E_{i j}=1 \Rightarrow F_{i(n-j)}$ $=0 \forall(i, j)$ (i.e. we do not interpolate at a discontinuity). Suppose further that one of the matrices $E, F$ is quasi-Hermite and the other has no supported odd blocks. Then the I.P. $(E, F, x)$ is poised for any $x$.

Proof. Suppose $E$ is quasi-Hermite and $b(F)=0$. We shall assume the I.P. $(E, F, x)$ is not poised and reach a contradiction. Then $\exists$ nonzero $f \in \zeta_{0}(F, x)$ satisfying (3.3).

Choose $r, k, l, 0<r<n, 0<k<l<m$, such that $f\left(x_{k}\right)^{-}=f\left(x_{l}\right)^{+}=0, f$ has exact degree $r$ on $\left(x_{k}, x_{l}\right)$ and $f$ is oscillating in $\left(x_{k}, x_{l}\right)$, i.e. $f$ does not vanish on any nontrivial interval in $\left(x_{k}, x_{l}\right)$. Let $\hat{F}=F\left(\begin{array}{c}k, \ldots, l \\ n-r, \ldots, n\end{array}\right)$.

Then for $\hat{x}=\left(x_{k}, \ldots, x_{l}\right), \exists$ nonzero $g \in \zeta_{0}(\hat{F}, \hat{x})$ with $g=f$ on $\left(x_{k}, x_{l}\right)$. Also $b(F)=0$. So by Corollary 2.1 ,

$$
Z(g)<\sum_{i=k}^{l} \sum_{j=0}^{r} \hat{F}_{i j}-(r+1) \leqslant \sum_{i=k+1}^{l-1} \sum_{j=0}^{r} E_{i j},
$$

by the Pólya conditions.

But $g^{(j)}\left(x_{i}\right)=0 \forall(i, j)$ with $E_{i j}=1$, and since all zeros in $\left(x_{k}, x_{l}\right)$ are isolated, we have $Z(g) \geqslant \sum_{i=k+1}^{l-1} \sum_{j=0}^{r} E_{i j}$, which gives the required contradiction.

The result is therefore true if $E$ is quasi-Hermite and $b(F)=0$. It follows from Theorem 3.1 that it is also true if $F$ is quasi-Hermite and $b(E)=0$.

4. The cardinal interpolation problem $(E, F, x)$. We now consider the C.I.P. $(E, F, x)$ as in (1.2). We again assume condition (3.2) but instead of (3.1) we assume the weaker condition,

$$
E_{0 j}=1 \Rightarrow F_{0(n-j)}=0, \quad j=0, \ldots, n,
$$

i.e. at the integers we do not interpolate at a discontinuity.

Now let $J=\left\{j ; E_{0 j}=F_{0(n-j)}=0\right\}$ and put $d=|J|$. 
We define matrices $E^{1}=\left\|E_{i j}^{1}\right\|_{i=0}^{m}{ }_{j=0}^{n}, F^{1}=\left\|F_{i j}^{1}\right\|_{i=0 j=0}^{m}, E^{2}=\left\|E_{i j}^{2}\right\|_{i=0}^{m} j_{j=0}^{n}$ and $F^{2}=\left\|F_{i j}^{2}\right\|_{i=0}^{m}{ }_{j=0}^{n}$ by

$$
\begin{aligned}
& E_{i j}^{1}= \begin{cases}1, & \text { if } i=0 \text { and } j \in J, \\
E_{i j}, & \text { otherwise, }\end{cases} \\
& F_{i j}^{1}= \begin{cases}1, & \text { if } i=m \text { and } n-j \in J, \\
F_{i j}, & \text { otherwise, }\end{cases} \\
& E_{i j}^{2}= \begin{cases}1, & \text { if } i=m \text { and } j \in J, \\
E_{i j}, & \text { otherwise, }\end{cases}
\end{aligned}
$$

and

$$
F_{i j}^{2}= \begin{cases}1, & \text { if } i=0 \text { and } n-j \in J \\ F_{i j}, & \text { otherwise. }\end{cases}
$$

THEOREM 4.1. If the I.P. $\left(E^{1}, F^{1}, x\right)$ and the I.P. $\left(E^{2}, F^{2}, x\right)$ are both poised, then for any choice of $\left\{y^{(i, j)}\right\}$ the solutions of (1.2) form a manifold of dimension $d$.

Proof. Clearly the elements $g \in \zeta(F, x)$ which satisfy

$$
\begin{aligned}
g^{(j)}\left(x_{i}\right)=y_{0}^{(i, j)} & \text { for } i=0, \ldots, m-1, j=0, \ldots, n, E_{i j}=1, \\
g^{(j)}(1)=y_{1}^{(0, j)} & \text { for } j=0, \ldots, n, E_{0 j}=1,
\end{aligned}
$$

form a manifold of dimension $d$. But any such $g$ can be uniquely extended to a solution of (1.2) and the result follows.

Henceforward we shall assume the I.P. $\left(E^{1}, F^{1}, x\right)$ and the I.P. $\left(E^{2}, F^{2}, x\right)$ are both poised. We let $e^{0}=e^{0}(E, F, x)$ denote the $d$-dimensional space comprising all solutions of (1.2) when all sequences $y^{(i, j)}$ are zero. We note that there is a nonsingular matrix $C=\left\|C_{\mu \nu}\right\|_{\mu, \nu \in J}$ such that for any $f \in \dot{e}^{0}$,

$$
f^{(\mu)}(1)=C_{\mu \nu} f^{(\nu)}(0), \quad \mu, \nu \in J .
$$

For the case of cardinal Hermite interpolation we see from Theorem 4.1 that $e^{0}$ has dimension $n+1-2 r$. In [10] Lipow and Schoenberg construct a basis for $\bigodot^{0}$ comprising what they term eigensplines, and then use this basis to prove that the C.I.P. is poised. Their work has been generalised by Micchelli [14] and the work of this section is a direct generalisation of $\$ 2$ of [14].

We call any $f$ in $\bigodot^{0}$ an eigenspline for the C.I.P. $(E, F, x)$ with eigenvalue $\lambda$ if

$$
f(x+1)=\lambda f(x), \quad \forall x \in \mathbf{R} .
$$

Clearly the eigenvalues of the C.I.P. $(E, F, x)$ are precisely the eigenvalues of the matrix $C$.

THEOREM 4.2. If the C.I.P. $(E, F, x)$ has $d$ distinct eigenvalues, then it is poised iff no eigenvalue lies on the unit circle.

Proof. If there is an eigenvalue on the unit circle, then there is a bounded eigenspline and so any solution of (1.2) of power growth is not unique and so the C.I.P. $(E, F, x)$ is not poised. 
We assume, then, that there are $d$ distinct eigenvalues not on the unit circle and show that the C.I.P. $(E, F, x)$ is poised. Take any $\gamma \geqslant 0$. Since the eigensplines span $\mathrm{C}^{0}$, there is no nonzero $f \in \mathrm{C}^{0}$ satisfying

$$
f(x)=O\left(|x|^{\gamma}\right) \text { as } x \rightarrow \pm \infty \text {. }
$$

Now for any $(k, l)$ with $F_{k l}=1$ and $0 \leqslant k<m$, we shall construct a function $L_{(k, l)} \in \mathcal{C}(F, x)$ satisfying

$$
L_{(k, l)}\left(\nu+x_{i}\right)=\delta_{0 \nu} \delta_{i k} \delta_{j l}
$$

Let $\lambda_{i}$ and $f_{i}, i=1, \ldots, d$, denote the corresponding eigenvalues and eigensplines. Then write

$$
L_{(k, l)}(x)= \begin{cases}\sum_{\left|\lambda_{i}\right|>1} c_{i} f_{i}(x), & x<-1, \\ p(x+1), & -1<x<0, \\ q(x), & 0<x<1, \\ \sum_{\left|\lambda_{i}\right|<1} c_{i} f_{i}(x), & x>1,\end{cases}
$$

where $c_{i}, i=1, \ldots, d$, are constants and $p, q \in \zeta(F, x)$. For (4.4) to be satisfied, we must solve $3 d+2 \sum_{i=0}^{m} \sum_{j=0}^{n} E_{i j}$ nonhomogeneous linear equations in the same number of unknowns. If there were no solutions, then there would be a nonzero solution to the homogeneous problem and thus a nonzero bounded element of $e^{0}$. Since this cannot happen, we conclude that we can construct $L_{(k, l)}$ of form (4.5) satisfying (4.4). From (4.5) we see that $L_{(k, l)}$ decays exponentially as $x \rightarrow \pm \infty$. If the sequences $\left\{y^{(i, j)}\right\}$ satisfy $y_{\nu}^{(i, j)}=O\left(|\nu|^{\gamma}\right)$ as $\nu \rightarrow \pm \infty$, then (1.2) is satisfied by

$$
f(x)=\sum_{\nu=-\infty}^{\infty} \sum_{(i, j)} y_{\nu}^{(i, j)} L_{(i, j)}(x-\nu)
$$

and this solution satisfies (4.3). If $g$ is another solution of (1.2) satisfying (4.3), then $f-g \in e^{0}$ satisfies (4.3) and so $f=g$.

We say the C.I.P. $(E, F, x)$ is symmetric if for $i=0, \ldots, m, j=0, \ldots, n$,

$$
E_{i j}=E_{(m-i) j}, \quad F_{i j}=F_{(m-i) j} \quad \text { and } \quad x_{i}=1-x_{m-i} .
$$

Thus the problem is invariant under the transformation $x \rightarrow 1-x$ and so $\lambda$ is an eigenvalue iff $\lambda^{-1}$ is an eigenvalue. This immediately gives us the following result.

Corollary 4.1. Suppose the C.I.P. $(E, F, x)$ is symmetric and has $d$ distinct real eigenvalues. Then it is poised iff an even number of eigenvalues are positive and an even number negative.

We also note that the duality between $E$ and $F$ extends to the following result concerning eigenvalues.

THEOREM 4.3. The C.I.P. $(E, F, x)$ has eigenvalue $\lambda$ iff the C.I.P. $(F, E, x)$ has eigenvalue $\lambda^{-1}$. 
Proof. Let $\left\{\left(i_{r}, j_{r}\right) ; r=1, \ldots, M(E)+d\right\}$ denote the elements of $\{(i, j) ; 0 \leqslant i$ $\left.<m, F_{i j}^{2}=1\right\}$ and $\left\{\left(k_{s}, l_{s}\right) ; s=1, \ldots, M(E)+d\right\}$ denote the elements of $\{(i, j)$; $\left.0<i \leqslant m, E_{i j}^{2}=1\right\}$ with the ordering as in the proof of Theorem 3.1. As before we let

$$
A=\left\|\frac{\left(x_{k_{s}}-x_{i_{r}}\right)_{+}^{n-j_{r}-l_{s}}}{\left(n-j_{r}-l_{s}\right) !}\right\|_{s, r=1}^{M(E)+d} .
$$

Let $n_{1}, \ldots, n_{d}$ denote the elements of $J$ in increasing order. Let $\left(0, n-n_{t}\right)=$ $\left(i_{r_{t}}, j_{r_{t}}\right)$ and $\left(m, n_{t}\right)=\left(k_{s_{t}}, l_{s_{t}}\right), t=1, \ldots, d$. Finally let

$$
B=A^{-1}\left(\begin{array}{c}
r_{1}, \ldots, r_{d} \\
s_{1}, \ldots, s_{d}
\end{array}\right) \text {. }
$$

Then for any $f \in \mathfrak{e}^{0}$ we have

$$
f^{\left(n_{i}\right)}(0)=B_{i j} f^{\left(n_{1}\right)}(1), \quad i, j=1, \ldots, d .
$$

Thus $\lambda$ is an eigenvalue of the C.I.P. $(E, F, x)$ iff $\lambda^{-1}$ is an eigenvalue of $B$. The result then follows as in the proof of Theorem 3.1.

Thus to determine whether the C.I.P. $(E, F, x)$ is poised, we must examine the eigenvalues of the matrix $C$ defined by (4.2). We recall that a square matrix is called totally positive if all its minors are nonnegative, and called strictly totally positive if all its minors are strictly positive. It is shown by Gantmacher and Krein (see [5, p. 105]) that an $N \times N$ oscillation matrix has $N$ distinct positive eigenvalues. This result has been generalised by Karlin and Pinkus [9] to the following.

Theorem 4.4 (Karlin AND Pinkus). For $N \geqslant 1$ and $\rho=0, \ldots, N$, define $I^{(\rho)}:=\left\|\varepsilon_{i} \delta_{i j}\right\|_{i, j=1}^{N}$, where $\varepsilon_{i}=-1$ for $i \leqslant \rho$ and $\varepsilon_{i}=1$ for $i>\rho$. Then if $A=$ $\left\|A_{i j}\right\|_{i, j=1}^{N}$ is an oscillation matrix, $\operatorname{det}\left(A-\lambda I^{(\rho)}\right)$ has $\rho$ distinct negative and $N-\rho$ distinct positive zeros.

For the case of cardinal Hermite interpolation, Lipow and Schoenberg show in [10] that $(-1)^{r} C$ is an oscillation matrix and thus $C$ has $d$ distinct eigenvalues of sign $(-1)^{r}$. Following Micchelli [14], we shall show that under certain conditions the matrix $\tilde{C}:=\left\|\left|C_{\rho \nu}\right|\right\|_{\mu, \nu \in J}$ is an oscillation matrix, and then apply Theorem 4.4 to examine, under certain conditions, the eigenvalues of $C$.

We now state conditions on $(E, F)$ that we shall assume throughout the rest of this paper. We shall not interpolate at a discontinuity, i.e. we assume

$$
E_{i j}=1 \Rightarrow F_{i(n-j)}=0 \text {. }
$$

We assume further that $E$ is quasi-Hermite and $\hat{F}=\left\|\hat{F}_{i j}\right\|_{i=0 j=0}^{m}$ has no supported odd blocks, where

$$
\hat{F}_{i j}= \begin{cases}1, & \text { if } i=0 \text { or } m, \text { and } E_{i(n-j)}=0, \\ F_{i j}, & \text { otherwise. }\end{cases}
$$

Also we assume $\left(E^{1}, F^{1}\right)$ and $\left(E^{2}, F^{2}\right)$ satisfy the Pólya conditions. Thus by Theorem 3.2, the I.P. $\left(E^{1}, F^{1}, x\right)$ and the I.P. $\left(E^{2}, F^{2}, x\right)$ are both poised. Now 
these Pólya conditions imply that

$$
\sum_{i=0}^{m-1} \sum_{j=0}^{r} E_{i j} \geqslant \sum_{i=0}^{m-1} \sum_{j=0}^{r} F_{i(n-j)}, \quad r=0, \ldots, n-1 .
$$

We shall make the stronger assumption that

$$
\sum_{i=0}^{m-1} \sum_{j=0}^{r} E_{i j}>\sum_{i=0}^{m-1} \sum_{j=0}^{r} F_{i(n-j)}, \quad r=0, \ldots, n-1 .
$$

For the case $0<i<m \Rightarrow F_{i j}=0$, conditions (4.6) are called the strong Pólya conditions on $E$.

Of course by duality, for any result we deduce under the above assumptions, there is a corresponding result when the conditions on $E$ and $F$ are interchanged.

THEOREM 4.5. (i) All the principal minors of $C$ are nonzero, i.e.

$$
\operatorname{det} C\left(\begin{array}{l}
\mu_{1}, \ldots, \mu_{l} \\
\mu_{1}, \ldots, \mu_{l}
\end{array}\right) \neq 0 \text { for any distinct } \mu_{1}, \ldots, \mu_{l} \in J \text {. }
$$

(ii)

$$
\operatorname{sgn} C_{\mu \nu}=(-1)^{\sigma_{\mu}+\tau_{\nu}}, \quad \text { where } \sigma_{\mu}=\sum_{j>\mu} E_{0 j}, \tau_{\nu}=\sum_{j>\nu} F_{0(n-j)}
$$

(iii) If

$$
\operatorname{det} C\left(\begin{array}{c}
\mu_{1}, \ldots, \mu_{l} \\
\nu_{1}, \ldots, \nu_{l}
\end{array}\right) \neq 0 \text { for } \mu_{1}<\cdots<\mu_{l}, \nu_{1}<\cdots<\nu_{l}
$$

then

$$
\operatorname{sgn} \operatorname{det} C\left(\begin{array}{c}
\mu_{1}, \ldots, \mu_{l} \\
\nu_{1}, \ldots, \nu_{l}
\end{array}\right)=\operatorname{sgn} \prod_{i=1}^{l} C_{\mu_{i} \nu_{i}}
$$

We first prove a lemma. Let $P$ and $Q$ be any nonempty subsets of $J$ with $|P|+|Q|=d$. We define $G=G(P, Q)=\left\|G_{i j}\right\|_{i=0 j=0}^{m} \quad$ and $H=H(P, Q)=$ $\left\|H_{i j}\right\|_{i=0 j=0}^{m}$ by

$$
G_{i j}= \begin{cases}1, & \text { if } i=0 \text { and } j \in P \\ 1, & \text { if } i=m \text { and } j \in Q \\ E_{i j}, & \text { elsewhere }\end{cases}
$$

and

$$
H_{i j}= \begin{cases}1, & \text { if } i=0 \text { and } n-j \in J-P, \\ 1, & \text { if } i=m \text { and } n-j \in J-Q \\ F_{i j}, & \text { elsewhere. }\end{cases}
$$


Lemma 4.1. Suppose the I.P. $(G, H, x)$ is poised and that a nonzero $f \in \zeta(H, x)$ satisfies

$$
f^{(j)}\left(x_{i}\right)=0 \quad \forall(i, j) \quad \text { with } G_{i j}=1 \text {, except } i=m, j=\max _{q \in Q} q .
$$

Then $f$ is oscillating and has exact degree $n$.

Proof. Take $f$ as above and choose $k, l, 0 \leqslant l \leqslant m$, such that $f\left(x_{k}\right)^{--}=f\left(x_{l}\right)^{+}=0$ and $f$ is oscillating in $\left(x_{k}, x_{l}\right)$. We cannot have $k>0$ since the I.P. $\left(E^{1}, F^{1}, x\right)$ is poised and we cannot have $l<m$ since the I.P. $\left(E^{2}, F^{2}, x\right)$ is poised. Thus $f$ is oscillating.

Suppose $f$ has exact degree $r$. Since the I.P. $(G, H, x)$ is poised we must have $r \geqslant \max _{q \in Q} q$. Then

$$
\begin{aligned}
& M\left(G\left(\begin{array}{c}
0, \ldots, m \\
r+1, \ldots, n
\end{array}\right)\right)-M\left(H\left(\begin{array}{c}
0, \ldots, m \\
r+1, \ldots, n
\end{array}\right)\right) \\
& \quad \leqslant M\left(E\left(\begin{array}{c}
0, \ldots, m \\
r+1, \ldots, n
\end{array}\right)\right)-M\left(F\left(\begin{array}{c}
0, \ldots, m \\
r+1, \ldots, n
\end{array}\right)\right)
\end{aligned}
$$

and so for $r<n$ we have by (4.6)

$$
\begin{aligned}
M\left(G\left(\begin{array}{l}
0, \ldots, m \\
0, \ldots, r
\end{array}\right)\right)-M\left(H\left(\begin{array}{l}
0, \ldots, m \\
0, \ldots, r
\end{array}\right)\right) \\
\quad \geqslant M\left(E\left(\begin{array}{l}
0, \ldots, m \\
0, \ldots, r
\end{array}\right)\right)-M\left(F\left(\begin{array}{l}
0, \ldots, m \\
0, \ldots, r
\end{array}\right)\right)>0 .
\end{aligned}
$$

But by Corollary 2.1,

$$
Z(f)<\sum_{i=0}^{m} \sum_{j=0}^{n} H_{i j}+1-(r+1) \leqslant \sum_{i=1}^{m-1} \sum_{j=0}^{r} G_{i j}
$$

by (4.8) which contradicts (4.7). So $r=n$.

ProOF OF THEOREM 4.5. We first note that

$$
\operatorname{det} C\left(\begin{array}{c}
\mu_{1}, \ldots, \mu_{l} \\
\nu_{1}, \ldots, \nu_{l}
\end{array}\right) \neq 0
$$

iff the I.P. $(G(P, Q), H(P, Q), x)$ is poised, where $P=J-\left\{\nu_{1}, \ldots, \nu_{l}\right\}, Q=$ $\left\{\mu_{1}, \ldots, \mu_{l}\right\}$.

If $\nu_{i}=\mu_{i}, i=1, \ldots, l$, we have $(G(P, Q), H(P, Q))$ satisfies the Pólya conditions because $\left(E^{1}, F^{1}\right)$ and $\left(E^{2}, F^{2}\right)$ do. This gives (i).

Now for $\nu \in J$, let $p_{\nu}$ denote the unique element of $\zeta(F, x)$ satisfying

$$
p_{\nu}^{(j)}\left(x_{i}\right)=0, \quad \text { when } E_{i j}=1,
$$

and

$$
p_{\nu}^{(j)}(0)=\delta_{j \nu} \quad \text { for } j \in J
$$

We see from (4.2) that

$$
C_{\mu \nu}=p_{\nu}^{(\mu)}(1) \quad \text { for } \mu, \nu \in J \text {. }
$$


By Lemma 4.1, with $P=J-\{\nu\}, Q=\{\nu\}$, we see that $p_{\nu}$ is oscillating and has exact degree $n$. We may therefore apply Theorem 2.1 to give (ii).

We prove (iii) by induction on $l$. It is clearly true for $l=1$. Assume it holds for $l$ - 1. Suppose

$$
\operatorname{det} C\left(\begin{array}{c}
\mu_{1}, \ldots, \mu_{l} \\
\nu_{1}, \ldots, \nu_{l}
\end{array}\right) \neq 0, \quad \mu_{1}<\cdots<\mu_{l}, \nu_{1}<\cdots<\nu_{l},
$$

and define

$$
p(x)=\left|\begin{array}{ccc}
C_{\mu_{1} \nu_{1}} & \cdots & C_{\mu_{1} \nu_{l}} \\
\vdots & & \vdots \\
C_{\mu_{l-1} \nu_{1}} & \cdots & C_{\mu_{l-1} \nu_{l}} \\
p_{\nu_{1}}(x) & \cdots & p_{\nu_{l}}(x)
\end{array}\right|
$$

We may then apply Lemma 4.1 with $P=J-\left\{\nu_{1}, \ldots, \nu_{l}\right\}, Q=\left\{\mu_{1}, \ldots, \mu_{l}\right\}$ to show that $p$ is oscillating and has exact degree $n$. It then follows from Theorem 2.1 and (ii) that

$$
\operatorname{sgn} p^{\left(\nu_{l}\right)}(0) p^{\left(\mu_{l}\right)}(1)=\operatorname{sgn} C_{\mu_{l} \nu_{i}}
$$

Since

$$
\operatorname{det} C\left(\begin{array}{c}
\mu_{1}, \ldots, \mu_{l-1} \\
\nu_{1}, \ldots, \nu_{l-1}
\end{array}\right)=p^{\left(\nu_{l}\right)}(0) \neq 0
$$

and

$$
\operatorname{det} C\left(\begin{array}{c}
\mu_{1}, \ldots, \mu_{l} \\
\nu_{1}, \ldots, \nu_{l}
\end{array}\right)=p^{(\mu)}(1)
$$

we may apply the inductive hypothesis to give (iii) for $l$.

COROLlaRy 4.2. The matrix $\tilde{C}=\left\|\left|C_{\mu \nu}\right|\right\|_{\mu, \nu \in J}$ is an oscillation matrix.

Proof. From Theorem 4.5 we see $\tilde{C}$ is a totally positive nonsingular matrix and $\tilde{C}_{\mu \nu}>0$ for all $\mu, \nu \in J$. It follows from a result of Gantmacher and Krein [5, p. 105] that $\tilde{C}$ is an oscillation matrix.

THEOREM 4.6. If $J=\left\{j_{1}, \ldots, j_{d}\right\}$ with $j_{1}<j_{2}<\cdots<j_{d}$, suppose there are numbers $\rho, \eta$ with $0 \leqslant \rho \leqslant d$ such that for $k=1, \ldots, d$,

$$
j_{k}+k+n+d+\eta \text { is } \begin{cases}\text { even } & \text { if } k \leqslant \rho, \\ \text { odd } & \text { if } k>\rho .\end{cases}
$$

Then the C.I.P. $(E, F, x)$ has $\rho$ distinct eigenvalues of sign $(-1)^{\eta}$ and $d-\rho$ distinct eigenvalues of sign $(-1)^{\eta+1}$.

Proof. The eigenvalues of the C.I.P $(E, F, x)$ are the solutions of

$$
\operatorname{det}(C-\lambda I)=0 \text {. }
$$


Now for each $\mu \in J$, multiply row $\mu$ of $C-\lambda I$ by $(-1)^{\sigma_{\mu}}$ and column $\mu$ by $(-1)^{\tau_{\mu}}$. Then by Theorem 4.5(ii), equation (4.10) becomes

$$
\operatorname{det}(\tilde{C}-\lambda \tilde{I})=0
$$

where $\tilde{I}=\left\|(-1)^{\sigma_{\mu}+\tau_{\mu}} \delta_{\mu \nu}\right\|_{\mu, \nu \in J}$. But if $\mu=j_{k}, 1 \leqslant k \leqslant d$,

$$
j_{k}=\sum_{j<\mu} E_{0 j}+\sum_{j<\mu} F_{0(n-j)}+k-1
$$

and so

$$
\begin{aligned}
\sigma_{\mu}+\tau_{\mu} & =\sum_{j>\mu} E_{0 j}+\sum_{j>\mu} F_{0(n-j)}, \\
& =n+1-d-\sum_{j<\mu} E_{0 j}-\sum_{j<\mu} F_{0(n-j)} \\
& =n-d-j_{k}+k .
\end{aligned}
$$

Thus

$$
\eta+\sigma_{\mu}+\tau_{\mu} \text { is } \begin{cases}\text { even } & \text { if } k \leqslant \rho, \\ \text { odd } & \text { if } k>\rho .\end{cases}
$$

So from Corollary 4.2 and Theorem 4.4, equation (4.11) has $\rho$ distinct solutions of $\operatorname{sign}(-1)^{\eta}$ and $d-\rho$ distinct solutions of sign $(-1)^{\eta+1}$.

Corollary 4.3. If $J=\left\{j_{1}, \ldots, j_{d}\right\}$ with $j_{1}<j_{2}<\cdots<j_{d}$, suppose there is a number $\rho$ with $1 \leqslant \rho \leqslant d$ such that for $k=1, \ldots, d-1$,

$$
j_{k+1}-j_{k} \text { is } \begin{cases}\text { even } & \text { if } k=\rho, \\ \text { odd } & \text { if } k \neq \rho .\end{cases}
$$

If the C.I.P. $(E, F, x)$ is symmetric, then it is poised if and only if $d$ and $\rho$ are even.

Proof. Writing $j_{k}=j_{1}+\sum_{l=1}^{k-1}\left(j_{l+1}-j_{l}\right)$ gives for $k=1, \ldots, d$

$$
j_{k}+j_{1}+k-1 \text { is } \begin{cases}\text { even } & \text { if } k \leqslant \rho, \\ \text { odd } & \text { if } k>\rho .\end{cases}
$$

The result then follows from Theorem 4.6 and Corollary 4.1.

Finally we consider an example which satisfies the hypothesis required for Corollary 4.3 to be applied. We consider a symmetric C.I.P. $(E, F, x)$ satisfying the following properties.

For all $i, j, E_{i j}=1 \Rightarrow F_{i(n-j)}=0$.

If $0<i<m$, the following hold.

(a) There is a number $M_{i} \geqslant 0$ such that $E_{i j}=1$ iff $j<M_{i}$.

(b) If $\{(i, j)\}, j=k, \ldots, k+l-1$, is an odd block in $F$, then $k=0$.

(c) $\sum_{j=0}^{n} F_{i j} \leqslant \min \left(M_{i}, \sum_{j=0}^{n} E_{0 j}\right)$.

Finally, we have

$$
\begin{gathered}
\sum_{i=0}^{m-1} \sum_{j=0}^{r} E_{i j}>\sum_{i=0}^{m-1} \sum_{j=0}^{r} F_{i(n-j)}, \quad r=0, \ldots, n-1, \\
\sum_{i=0}^{m-1} \sum_{j=0}^{n} E_{i j}=\sum_{i=0}^{m-1} \sum_{j=0}^{n} F_{i j} .
\end{gathered}
$$


It is straightforward to verify that $\left(E^{1}, F^{1}, x\right)$ and $\left(E^{2}, F^{2}, x\right)$ satisfy the Pólya conditions and so $(E, F, x)$ satisfies all the hypotheses required for the application of Corollary 4.3. This example includes all the particular examples considered in [14].

To give a more specific example not considered in [14] we consider the following symmetric C.I.P. $(E, F, x)$ with $m \geqslant 2, n \geqslant 5$.

For $i=1, \ldots, m-1, E_{i j}=1$ iff $j=0$ or 1 and $F_{i j}=1$ iff $j=1$ or 2. Also $E_{01}=F_{01}=1,2 \leqslant \sum_{j=0}^{n} E_{0 j}=\sum_{j=0}^{n} F_{0 j}<2 m$, and $E_{0 j}=1 \Rightarrow F_{0(n-j)}=0$. It is easily checked that this is a special case of the previous example.

\section{REFERENCES}

1. K. Atkinson and A. Sharma, A partial characterization of poised Hermite-Birkhoff interpolation problems, SIAM J. Numer. Anal. 6 (1969), 230-235.

2. G. D. Birkhoff, General mean value and remainder theorems, Trans. Amer. Math. Soc. 7 (1906), 107-136.

3. C. de Boor and I. J. Schoenberg, Cardinal interpolation and spline functions. VIII. The Budan-Fourier theorem for splines and applications, Spline Functions (Proc. Internat. Sympos., Karlsruhe, 1975), Lecture Notes in Math., vol. 501, Springer-Verlag, Berlin and New York, 1976, pp. 1-79.

4. D. R. Ferguson, Sign changes and minimal support properties of Hermite-Birkhoff splines with compact support, SIAM J. Numer. Anal. 11 (1974), 769-779.

5. F. R. Gantmacher, The theory of matrices, Vol. II, Chelsea, New York, 1964.

6. T. N. T. Goodman and S. L. Lee, A remainder formula and limits of cardinal spline interpolants, Trans. Amer. Math. Soc. 271 (1982), 469-483.

7. K. Jetter, Birkhoff interpolation by splines, Approximation Theory. II, (G. G. Lorentz, C. K. Chui and L. L. Schumaker, Editors), Academic Press, New York, 1976, pp. 405-410.

8. S. Karlin and C. Micchelli, The fundamental theorem of algebra for monosplines satisfying boundary conditions, Israel J. Math. 11 (1972), 405-451.

9. S. Karlin and A. Pinkus, Oscillation properties of generalised characteristic polynomials for totally positive and positive definite matrices, Linear Algebra Appl. 8 (1974), 103-111.

10. P. R. Lipow and I. J. Schoenberg, Cardinal interpolation and spline functions. III. Cardinal Hermite interpolation, Linear Algebra Appl. 6 (1973), 273-304.

11. G. G. Lorentz, Zeros of splines and Birkhoff's kernel, Math. Z. 142 (1975), 173-180.

12. A. A. Melkman, The Budan-Fourier theorem for splines, Israel J. Math. 19 (1974), 256-263.

13. __ Hermite-Birkhoff interpolation by splines, J. Approx. Theory 19 (1977), 259-279.

14. C. A. Micchelli, Oscillation matrices and cardinal spline interpolation, Studies in Spline Functions and Approximation Theory, Academic Press, New York, 1976, pp. 163-201.

15. D. D. Pence, Hermite-Birkhoff interpolation and monotone approximation by splines, J. Approx. Theory 25 (1979), 248-257.

16. I. J. Schoenberg, On Hermite-Birkhoff interpolation, J. Math. Anal. Appl. 10 (1966), 538-543.

17. L. L. Schumaker, Towards a constructive theory of generalised spline functions, Spline Functions (Proc. Internat. Sympos., Karlsruhe, 1975), Lecture Notes in Math., vol. 501, Springer-Verlag, Berlin and New York, 1976, pp. 265-329.

Department of Mathematics, The University, Dundee DDl 4hN, Dundee, Scotland, United KINGDOM

School of Mathematical Sciences, University Science Malaysia, Penang, Malaysia 